\title{
Long-Term Adaptations to Aerobic-Anaerobic Physical Training in the Erythrocyte Membrane Fatty Acids Profile
}

\author{
Pablo J Iglesias ${ }^{1}$, Diego Muñoz ${ }^{1 *}$, Francisco Llerena ${ }^{2}$, Francisco J Grijota ${ }^{1}$, Ignacio Bartolome ${ }^{1}$ and \\ Marcos Maynar ${ }^{1}$
}

${ }^{1}$ Department of Physiology, University of Extremadura, Spain

${ }^{2}$ University of Avenue, Spain

*Corresponding author: Diego Muñoz, Sport Sciences Faculty, Department of Physiology, University of Extremadura, Avenida de la Universidad s/n, Cáceres (Cáceres), 10003, Spain, Tel: 0034927257460 (ext 57833), Fax: 0034927257461 , E-mail:diegomun@unex.es

\begin{abstract}
Aim: The aim of this survey is to examine the effects of physical training on erythrocyte membrane fatty acids percentages as well as to analyze cellular adaptations in young sportsmen.

Methods: Forty-four male volunteers participated: a group of 22 Trained players (TG) (age: $17.86 \pm 0.36$ years; weight: $69.86 \pm 4.53 \mathrm{Kg}$; height: $1.78 \pm 0.54 \mathrm{~m})$, with a regular football training plan of 10 hours/week, and a group of 22 Untrained participants (UG) (age: $18.23 \pm 0.49$ years; weight: $72.10 \pm 8.56 \mathrm{Kg}$; height: $1.68 \pm 0.39 \mathrm{~m}$ ). Gas chromatograph HP-5890 Series II was used to determine fatty acids concentrations.

Results: Higher levels were obtained in the TG group in C12:0 (0.31 \pm 0.35 vs. $0.03 \pm 0.04 \%, P<0.001)$ and in $\mathrm{n}-6 / \mathrm{n}-3$ index ( $8.74 \pm 5.20$ vs. $4.29 \pm 1.09, \mathrm{P}<0.001)$, and lower values in $\mathrm{C} 22: 6(3.00 \pm 3.51$ vs. $5.08 \pm 1.44 \%, \mathrm{P}<$ 0.01 ) in comparison with the UT group. Moreover, a correlation of these parameters and the Lipid Peroxidation index with training degree were observed.

Conclusion: 10 hours/week aerobic-anaerobic football training program produces $\mathrm{C} 12: 0, \mathrm{n}-6 / \mathrm{n}-3$ index, $\mathrm{C} 22: 6$ modifications in erythrocyte membrane.
\end{abstract}

\section{Keywords}

Erythrocytes, Lipid peroxidation, Fatty acids, Football, Exercise, Physiological adaptations

\section{Introduction}

Fatty acids are critical organic compounds involved in the synthesis of other lipids like phospholipids and glycolipids constituting lipid bilayer of all cell membranes, besides they are an important source of chemical energy [1]. A correlation between several fatty acids and health has been demonstrated [2]. High concentrations of saturated fatty acids can convey harmful effects, whereas unsaturated fatty acids may develop a protective role $[3,4]$. They are considered an attractive target to regulate the human diseases of obesity, diabetes, cancer, cardiovascular complications and inflammatory processes $[5,6]$.

Fatty acids half-life in adipose tissues is estimated to last 680 days, while erythrocyte half-life is of around 120 days. Therefore, to reflect long-term adaptations, erythrocytes are a more suitable organic matrix than plasma [7-11].

It is well established $[12,13]$ that physical exercise induces Reactive Oxygen Species (ROS) and free radicals production. This production is closely linked to with exercise intensity, being the highest concentrations in strenuous exercise [12].

An intrinsic property of ROS is a high-reactivity with several substances. Polyunsaturated Fatty Acids (PUFA) are strongly affected by this reactivity, and structures rich in PUFAs can consequently be damaged by ROS, like cell membranes and lipoproteins $[14,15]$.

Another important property of ROS and fatty acids interaction is an oxidative-chain-reaction; when fat- 
ty acids are oxidized, they become fatty acid radicals. These lipidic radicals are able to oxidize other neighboring molecules. This process is known as lipid peroxidation $[16,17]$. It has been pointed out that lipid peroxidation decreases erythrocyte membrane PUFA as a consequence of acute physical exercise and training [18].

It has been demonstrated that a consequence of endurance training is an increased tissue lipid concentrations as well as a modified lipid profile [19]. Furthermore, high-intensity exercise may raise plasma Monounsaturated Fatty Acids (MUFA) concentrations, whereas chronic-exercise adaptations seem to increase PUFA and $n-6$ fatty acids proportion. When tissues proportion of MUFA decreases by means of exercise, the amount of unsaturated fatty acids in human liver could decrease further, independently of nutrition [20].

Scientific evidences of training adaptations in the erythrocyte membrane of young population have not been found in the literature. However, the relationship between training and fatty acids behavior has been previously studied in female athletes, male water polo and adult football players [21] and it has been observed that long-term, intense physical training amends fatty acids profile, and, in the case of amateur boxers [22] causes significant modifications in saturated fatty acids.

A group of young football players participated in the present research. Football is one of the most practiced sports world wide. Numerous studies have described physical performance in recent men's football [23].

However, there is little evidence of this among young athletes. Football can be described as an aerobic-anaerobic modality where maximal efforts and recovery times are alternated [24]. Lipidic metabolism becomes critical in this sport in the low-intensity phases, when caloric expenditure is supplied by means of lipid oxidation [25].

This work aims to evaluate the long-term effect of aerobic-anaerobic exercise on basal erythrocyte fatty acid concentrations in both Trained (TG) and Untrained (UG) and participants, and to identify the possible relationship existing between the properties of the erythrocyte membrane and the degree of training.

\section{Material and Methods}

\section{Ethics guidelines}

The study was approved by the Ethics Committee of the University of Extremadura under the ethical guidelines of the Declaration of Helsinki of the World Medical Association (updated World Medical Assembly in Seoul 2008) for human research.

\section{Participants}

44 young males participated in this research; they were divided into two groups according to their fitness level. All participants had been living in the region of Cáceres, in Extremadura (Spain) for, at least, 24 months
Table 1: Trained and untrained anthropometric characteristics.

\begin{tabular}{|l|l|l|l|}
\hline & Trained (TG) & Untrained (UG) & $\boldsymbol{p}$ value \\
\hline Age (years) & $17.86 \pm 0.36$ & $18.23 \pm 0.49$ & 0.01 \\
\hline Weight $(\mathrm{kg})$ & $69.86 \pm 4.53$ & $72.10 \pm 8.56$ & 0.28 \\
\hline Height $(\mathrm{m})$ & $1.78 \pm 0.54$ & $1.68 \pm 0.39$ & 0.12 \\
\hline Fat $(\%)$ & $10.84 \pm 1.63$ & $14.88 \pm 6.23$ & 0.01 \\
\hline Muscle (\%) & $48.62 \pm 2.27$ & $45.55 \pm 6.82$ & 0.00 \\
\hline
\end{tabular}

before the beginning of the research. The area is a reduced region of Extremadura (Spain).

All participants were informed, and they accepted their voluntary participation and gave their informed written consent.

\section{Inclusion criteria}

For their inclusion in the study, the participants had to meet the following criteria: be a male, do not follow any special diet or take vitamin/mineral supplements, or specific supplementation, and do not have any injury or illness during the investigation period.

\section{Fitness level}

IPAQ questionnaire [26] was completed by every participant the week before the beginning of the research. According to the data obtained, they were classified in two different groups: The Trained Group (TG) and the Untrained Group (UG). TG IPAQ scores were 10 hours/ week of specific, planned exercise, and had, at least, 5 years' experiences in competition sport training. The UG participants performed physical activity for no more than 3 hours/week.

\section{Anthropometric characteristics}

Anthropometric characteristics of both groups are shown in Table 1. TG consisted of 22 football players (age: $17.86 \pm 0.36$ years; weight: $69.86 \pm 4.53 \mathrm{Kg}$; height: $1.78 \pm 0.54 \mathrm{~m}$ ) from a youth category belonging to group $\mathrm{V}$ of the National Honor Division. They were semi-professional football players with a regular training plan of 10 hours/week. All of them had been performing high level competitions and had training sessions for at least five years before the experimental period.

UG consisted of 22 youngsters (age: $18.23 \pm 0.49$ years; weight: $72.10 \pm 8.56 \mathrm{Kg}$; height: $1.68 \pm 0.39 \mathrm{~m}$ ) who had not followed a systematic training plan for at least the last 6 months. Their weekly physical activity was below 3 hours/week.

\section{Fatty acids intake}

For the sixth previous week to the end of the experimental period, the TG and the UG had not taken any specific supplement of vitamins, minerals or fatty acids.

For the 2 previous years, both groups had not followed any specific nutritional plan and all participants had been on a Mediterranean Diet (MD). It is noticeable that MD is a MUFA and PUFA rich-in diet. As both groups had been on an analogous diet, the proportion 
Table 2: Methods of calculation of SFA, UFA, desaturation and lipid peroxidation indexes.

\section{Index}

Saturated Fatty Acids (SFA)

Monounsaturated Fatty Acids (MUFAs)

Polyunsaturated Fatty Acids n-6 (n-6 PUFAs)

Polyunsaturated Fatty Acids n-3 (n-3 PUFAs)

Total Polyunsaturated Fatty Acids (PUFAs)

$\mathrm{n}-6 / \mathrm{n}-3$ Index

Desaturation index Delta 9

n-3 Delta 5 index

n-6 Delta 5 index

$\mathrm{n}-3$ Lipid peroxidation index

n-6 Lipid peroxidation index

of fatty acids should not vary among both groups, due to a similar nutrients intake.

\section{Experimental design}

The tests were carried out before the start of the season, in July, after 3 months of inactivity of the trained group. Anthropometrics measurements and calculations were made under the guidelines of the Kin anthropometry Spanish Group [27]. To determine fat percentage Yuhasz equation was used [28]. Muscle percentage was determined from the calculation of muscle weight, by getting the difference between the total weight and other body weights: bone, fat and waste [28].

\section{Laboratory measurements}

Blood samples from the antecubital vein were extracted in $10 \mathrm{~mL}$ tubes with Ethylene Diamine Tetra Acetic Acid (EDTA), in rest conditions in both groups, after a 12 hours fasting period. The blood samples were immediately centrifuged for 10 minutes at $3000 \mathrm{rpm}$. Then erythrocytes were washed with $0.9 \%$ sodium chloride $(\mathrm{NaCl})$ three times. Fatty acid concentrations were determined in erythrocytes using the technique described by Lepage and Roy [29]. A gas chromatograph HP-5890 Series II was used, with a Flame Ionization Detector (FID). The column used was a BP $\times 70$ capillary column $50 \mathrm{~m} \times$ $0.22 \mathrm{~mm}$ I.D., film thickness $0.25 \mu \mathrm{m}$, Cromlab (Barcelona. Spain). The initial oven temperature was set at 170 ${ }^{\circ} \mathrm{C}$ and held for $15.0 \mathrm{~min}$. It was gradually increased to $190{ }^{\circ} \mathrm{C}$ for $15.0 \mathrm{~min}$ at a rate of $3^{\circ} \mathrm{C} / \mathrm{min}$ and to $245^{\circ} \mathrm{C}$ at $3{ }^{\circ} \mathrm{C} / \mathrm{min}$. The final temperature was maintained for $30.0 \mathrm{~min}$. The inert gas used was Helium ( $\mathrm{He}$ ) at a flow rate of $1.0 \mathrm{~mL} / \mathrm{min}$. The injector was used in split less mode, its temperature set at $300{ }^{\circ} \mathrm{C}$ and a purge flow of $6 \mathrm{~mL} / \mathrm{min}$ was applied $0.5 \mathrm{~min}$ after the injection. FID was used and set at $250{ }^{\circ} \mathrm{C}$.

The identification of fatty acids was carried out by comparing retention times of the methyl derivatives of the fatty acids studied, with the fatty acid patterns in the same chromatographic conditions and using retention parameters relative to the internal standard. The internal pattern chosen was heptadecanoic acid, because it is a substance similar to those analyzed and it is well located in the chromatogram, which did not interfere

\section{Molecules}

C12:0+C14:0+C16:0+C18:0+C24:0

C16:1+C18:1C+C18:1T+C24:1

C18:2:6+C18:3:6+C20:3:6+C20:4:6

C18:3:3+C20:5:3+C22:5:3+C22:6:3

$\mathrm{n}-6$ PUFAs $+\mathrm{n}-3$ PUFAs

$n-6$ PUFAs/n-3 PUFAs

C18:1 and C18:0 ratio

$\mathrm{C} 22: 6: 3$ and $\mathrm{C} 22: 5: 3$ ratio ( $\mathrm{n}-3$ family)

$\mathrm{C} 20: 4: 6$ and $\mathrm{C} 20: 3: 6$ ratio ( $\mathrm{n}-6$ family)

C22:6:3/C12:0

C20:4:6/C12:0

with other peaks in the sample. Fatty acids results are expressed as a percentage of the total fatty acids.

After obtaining fatty acids values, fatty acid indexes shown in Table 2 were calculated according to the method conducted by Halliwell and Gutteridge [30].

$\mathrm{N}-6-P U F A / n-3-P U F A$ index shows the coeficient between total fatty acids $n-6$ and $n-3$, and it has a relation with membrane stability and with inflammation and autoimmunity $[22,31]$.

$\Delta$ Index refers to the action of the desaturases, enzymes responsible for fatty acid desaturation making double bonds. Moreover, the Lipid Peroxidation (LP) index informs on the breakdown of long-chain fatty acids into short-chain fatty acids as a consequence of the oxidative chain reaction [30].

\section{Data analysis}

The software IBM SPSS 19.0 for windows was used for data analysis and treatment. The Data are reported as mean \pm standard deviation. Kolmogorov Smirnov test was used to ensure a normal data distribution. A comparison between the behaviors of the variables with the two populations was performed using the significance student's t-test test to compare the means in independent groups. The differences were considered statistically significant when $p<0.05$. Pearson's correlation coefficients were calculated between variables and performance status. A value of $p<0.05$ was used to determine statistical significance.

\section{Results}

Differences between the TG and the UG related to age, weight, height, fat percentage and muscular percentage are showed in Table 1.

It can be observed differences in fat and muscular percentage variables $(p<0.05)$. In this sense, the TG has lower fat percentage and higher muscular percentage in comparison with the UG.

The concentrations found in SFA, MUFA and PUFA percentages are showed in Table 3 . The results show a difference $(p<0.01)$ in the short chain fatty acid lauric acid (12:0) between the TG and the UG, with much higher concentrations in the TG. 
Table 3: SFA, MUFA, PUFA $n-6$ and $n-3$ percentage values in trained and untrained participants (results are expressed as a percentage of the total fatty acids).

\begin{tabular}{|l|l|l|l|}
\hline & Trained & Untrained & $\boldsymbol{p}$ value \\
\hline C12:0 & $0.31 \pm 0.35$ & $0.03 \pm 0.04$ & 0.00 \\
\hline C14:0 & $0.16 \pm 0.08$ & $0.43 \pm 1.02$ & 0.22 \\
\hline C16:0 & $20.76 \pm 11.47$ & $18.34 \pm 0.85$ & 0.33 \\
\hline C18:0 & $16.19 \pm 4.46$ & $16.24 \pm 0.92$ & 0.96 \\
\hline C24:0 & $6.35 \pm 2.02$ & $7.01 \pm 1.67$ & 0.24 \\
\hline C16:1 & $0.17 \pm 0.08$ & $0.21 \pm 0.09$ & 0.13 \\
\hline C18:1:C & $12.39 \pm 39$ & $11.40 \pm 0.80$ & 0.16 \\
\hline C18:1:T & $0.44 \pm 0.23$ & $0.53 \pm 0.39$ & 0.33 \\
\hline C24:1 & $6.85 \pm 0.73$ & $5.98 \pm 3.23$ & 0.22 \\
\hline C18:2:6 & $11.51 \pm 2.23$ & $10.62 \pm 1.18$ & 0.11 \\
\hline C18:3:6 & $0.08 \pm 0.07$ & $0.12 \pm 0.23$ & 0.53 \\
\hline C20:3:6 & $1.80 \pm 0.62$ & $1.74 \pm 0.47$ & 0.71 \\
\hline C20:4:6 & $18.31 \pm 3.35$ & $18.81 \pm 0.92$ & 0.51 \\
\hline C18:3:3 & $0.45 \pm 0.18$ & $0.47 \pm 0.04$ & 0.67 \\
\hline C20:5:3 & $0.88 \pm 1.21$ & $0.73 \pm 0.28$ & 0.60 \\
\hline C22:5:3 & $1.21 \pm 1.53$ & $1.39 \pm 0.45$ & 0.59 \\
\hline C22:6:3 & $3.00 \pm 3.51$ & $5.08 \pm 1.44$ & 0.01 \\
\hline SFA & $43.78 \pm 8.95$ & $42.06 \pm 1.29$ & 0.38 \\
\hline MUFA & $18.97 \pm 3.61$ & $18.98 \pm 0.87$ & 0.99 \\
\hline PUFA & $37.25 \pm 6.38$ & $38.96 \pm 1.03$ & 0.22 \\
\hline n-6 PUFA & $31.71 \pm 5.52$ & $31.29 \pm 1.29$ & 0.06 \\
\hline n-3 PUFA & $5.54 \pm 4.75$ & $7.67 \pm 1.65$ & 0.73 \\
\hline
\end{tabular}

Table 4: n-6 PUFA and LP PUFA/n-3 desaturation indexes in trained and untrained groups.

\begin{tabular}{|l|l|l|l|}
\hline & Trained & Untrained & $\boldsymbol{p}$ value \\
\hline n-6/n-3 Index & $8.74 \pm 5.20$ & $4.29 \pm 1.09$ & 0.00 \\
\hline Delta 9 Index & $1.94 \pm 5.81$ & $0.70 \pm 0.06$ & 0.32 \\
\hline Delta 5- n6 Index & $10.17 \pm 2.79$ & $10.81 \pm 2.57$ & 0.42 \\
\hline Delta 5- n3 Index & $2.47 \pm 4.72$ & $3.65 \pm 2.22$ & 0.73 \\
\hline LP 22:6:3 Index & $11.47 \pm 2.52$ & $66.64 \pm 2.74$ & 0.00 \\
\hline LP 20:4:6 Index & $76.54 \pm 30.90$ & $241.3 \pm 60.38$ & 0.00 \\
\hline
\end{tabular}

Differences $(p<0.05)$ in docosahexanoic acid (DHA, 22:6:3) were found, with higher concentrations in UG participants. Notably, TG had higher SFA concentrations, however they had lower concentrations in PUFA. $\mathrm{N}-6$ fatty acids showed higher percentages in the TG than in UG but without statistical significance.

The results obtained in $n-6 / n-3$ index, desaturation and LP are reflected in Table 4. Significant differences $(p$ $<0.01$ ) can be detected in $n-6 / n-3$ index between the TG and the UG, much higher levels being found in the TG. Furthermore, differences $(p<0.01)$ can be appreciated in LP index in relation with short chain fatty acids (12:0), still higher concentrations were found in the UT group.

The correlations between the different fatty acids and different indicators analyzed in this study with the training variable are presented in Table 5 and Table 6 . As it is shown in the tables, there is a high positive correlation between 12:0 $(p<0.01)$ and the level of training, oppositely, a negative correlation between 22:6 ( $p=$ 0.01 ) and the level of training has been found.

As it can be observed in Table 6, significant correlations were obtained in $n-6 / n-3$ and LP indexes; there is
Table 5: Training degree and fatty acids concentration correlations.

\begin{tabular}{|l|l|l|}
\hline & Pearson's correlation & $\boldsymbol{p}$ value \\
\hline C12:0 & 0.51 & 0.00 \\
\hline C14:0 & -0.19 & 00.21 \\
\hline C16:0 & 0.15 & 00.33 \\
\hline C18:0 & -0.01 & 00.96 \\
\hline C24:0 & -0.18 & 0.24 \\
\hline C16:1 & -0.23 & 0.13 \\
\hline C18:1C & 0.22 & 0.16 \\
\hline C18:1T & -0.15 & 0.33 \\
\hline C24:1 & -0.19 & 0.22 \\
\hline C18:2:6 & 0.25 & 0.11 \\
\hline C18:3:6 & -0.10 & 0.53 \\
\hline C20:3:6 & 0.06 & 0.70 \\
\hline C20:4:6 & -0.10 & 0.51 \\
\hline C18:3:3 & -0.06 & 0.69 \\
\hline C20:5:3 & 0.09 & 0.59 \\
\hline C22:5:3 & -0.08 & 0.60 \\
\hline C22:6:3 & -0.37 & 0.01 \\
\hline
\end{tabular}

Table 6: Correlations among training degree and delta, peroxidation, and $n-6 / n-3$ indexes.

\begin{tabular}{|l|l|l|}
\hline & Pearson's correlation & $p$ value \\
\hline SFA & 0.14 & 0.0 .38 \\
\hline MUFA & -0.00 & 10.00 \\
\hline PUFA & -0.19 & 00.22 \\
\hline n-6 PUFA & -0.29 & 0.06 \\
\hline n-3 PUFA & 0.05 & 0.73 \\
\hline n-6/n-3 Index & 0.52 & 0.00 \\
\hline Delta 9 & 0.15 & 0.32 \\
\hline Delta 5- n-6 & -0.13 & 0.42 \\
\hline Delta 5- n-3 & 0.05 & 0.73 \\
\hline LP 22:6:3 Index & -0.79 & 0.00 \\
\hline LP 20:4:6 Index & -0.66 & 0.00 \\
\hline
\end{tabular}

a high correlation between them and the training level. A positive relationship $(p<0.01)$ was obtained between the $n-6 / n-3$ index and training level. On the other hand, there was an inverse relationship $(p<0.01)$ between LP and training.

\section{Discussion}

The first aim of the present research was to evaluate the long-term influence of aerobic-anaerobic exercise on erythrocyte fatty acid composition. In the present survey acute exercise-induced effect has been avoid. On the one hand, because blood samples were extracted after three months of TG inactivity. On the other hand, because these samples were extracted in recovery conditions, without any previous acute exercise-induced effect.

One of the factors which may influence fatty acid composition is diet $[32,33]$. The effects of fatty acids intake on erythrocyte membrane are well documented [34]. That is why diet was partially-controlled in this research. Firstly, none of participants followed any specific nutritional plan and none of them took any mineral/vita$\mathrm{min} /$ fatty acids supplementation. Secondly, because the TG and the UG participants had been living in the same, 
reduced, geographic area and they had been following a similar Mediterranean Diet for at least two years before the blood extractions day. Consequently, the variability in the type of foods consumed and in the nutritional routines was minimal. Additionally, the TG had been physically inactive for the previous three months, and they had been following a maintenance caloric intake plan, in order to maintain an adequate fitness level for the following pre-season and to avoid increases in body weight. Accordingly, the nutritional plan of both groups was similar.

In this sense, the results of this study show that longterm, systematic aerobic-anaerobic physical exercise causes anthropometric changes, as shown in Table 1. The TG showed higher $(p<0.01)$ muscle $\%$ and lower ( $p$ $=0.01$ ) fat $\%$. This lower fat $\%$ and the higher muscle $\%$ in the TG could be linked to the increased metabolism of lipids as an energy source $[35,36]$. These results are consistent with those obtained in another study among males of similar age who were carrying out a football training program for 10 weeks, and obtained reductions in fat $\%$ by $4.72 \%$ [37].

It is well known that regular physical training induces changes in fatty acid composition. In this sense, Helge [33] observed differences in phospholipid fatty acids of muscular membranes after a 4 week training period. Andersson [38] reported modifications in several fatty acids as a consequence of a 10-week training period. This data manifests a real correlation between physical training and fatty acids composition. Experimentally, this research differs from the above mentioned surveys because this sport population had been practicing similar specific training for longer periods (more than 24 months). The modifications reported in this research could imply chronic biochemical adaptations as a consequence of the aerobic-anaerobic specific demands of football maintained for long periods of time.

Erythrocyte is recognized as a suitable matrix to research long-term physiological adaptations, in comparison to plasma [7-11]. Fatty acid composition of erythrocyte membranes carry out a very important role in the protection of cell integrity during strenuous exercise. These conditions can produce high stress in body cells, erythrocyte included, by means of Radical Oxygen Species (ROS). The metabolites produced during strenuous exercise forces cells to adapt their composition in order to overwhelm these situations and increase performance. When these stimulate are maintained for long periods, the human body develops physiological adaptations to prevent damage when practising sport (football in the case of these participants). Therefore, it can be stated that phospholipids and fatty acids play an important role in the biochemical adaptations of the cell membranes against exercise-induced damage.

The TG presented 12:0 higher $(p=0.00)$ levels and 22:6:3 lower $(p=0.01)$ values than the UT. No studies have been found that concern $12: 0$ and exercise relationship. However, 22:6:3 is considered as one of the essential fatty acids, and is directly linked to a blood pressure decrease [39].

The explanation of these results can be aided with previous reports. Arsic [21] made a comparative analysis of fatty acid profiles in erythrocyte membranes among female football and water polo players in both similar energetic exercise modalities (aerobic-anaerobic); they found a significantly higher proportion of oleic acid (18:1C) in the football group than in the control group. In anaerobic sports, such as boxing, predominant lower values of linoleic acid (18:2:6) were registered in comparison with those in the control group [22].

On the other hand, in sports like water polo, there was a decrease of stearic acid (18:0) and an increase of palmitic (16:0) and palmitoleic (16:1) acids among the sportsmen in comparison with those in the control and the football players groups [21].

These findings suggest that fatty acids composition among sportsmen varies according to the sport practiced and clear conclusions about fatty acids profiles and sports cannot be drawn, because it seems that each fatty acid showed a different response to each type of exercise.

This fact was especially noticeable among female football players, amending, in this case, oleic acid (18:1C) and MUFA, whereas in another study among professional football players, palmitoleic acid (16:1) was amended by Tepsic [40].

The results obtained in this study show higher values of total SFA and n-6 PUFA, and lower n-3 PUFA in the trained participants than in the UG (Table 3 ). Despite no significant differences were obtained, these results agree with those found in other studies where aerobic-anaerobic exercise was practiced, one among basketball players, with a similar response in SFA and $n-6$ PUFA [40], and among boxers with a similar trend in SFA [22]. So, if previous reports are taken into account, it can be stated that there is a clear change in SFA and $n-6$ PUFA in response to aerobic-anaerobic exercise. PUFA's plays an important role in protecting the organism [3]. Plasma n-3 PUFA reduces plasma triglycerides, resting heart rate and blood pressure and besides improving myocardial filling and efficiency, by reducing inflammation and improving the vascular function [41]. This data reveals a clear healthy benefit from aerobic-anaerobic exercise practice.

Furthermore, significant differences were obtained in the n-6/n-3 index, desaturation and Lipid Peroxidation (LP) indexes (Table 4) between the TG and the UG, being the highest $(p<0.01)$ values in the TG. Tepsic [22] found a greater $n-6 / n-3$ ratio in boxers in comparison with those in the control group; it was shown that a high $n-6 / n-3$ ratio modulates inflammation and autoimmu- 
nity processes. It has been recently demonstrated that high seric levels in $n-6 / n-3$ PUFA ratio induce health benefits while lower ratios might develop adverse effects [42]. In this sense obtained data might reflect possible exercise-induced adverse conditions in sportsmen.

However, significant differences were found in the LP indexes linked to short chain fatty acid (12:0) with even higher values in the trained males. Oxidative damage to membrane lipids is the most important factors in decreasing membrane fluidity [43]. The greatest damage caused by free radicals is PUFA peroxidation, which causes cellular membranes malfunction $[14,15]$.

The rise in 12:0 fatty acids in the TG could have two possible explanations. The first one might be due to a PUFA n3 $L P$, and this could be necessary to increase the erythrocyte membrane stiffness, which is submitted to great capillaries pressure during physical activity. The second explanation for this could be that the high intensity training carried out by football players might affect the cellular antioxidant system levels to protect them against ROS activity, a fact which has not been demonstrated.

The correlations between fatty acids and training are shown in Table 5 and Table 6. Obtained data show that $12: 0$ and $n-6 / n-3$ index have a direct proportional relationship with the training level, while 22:6:3, and LP index have an inversely proportional relationship, which might indicate a destruction of long chain fatty acids 22:6:3 and 20:4:6 as a result of the acceleration that takes place in lipid peroxidation among the TG participants due to exercise.

Due to the lack of estimation of parameters related to oxidative stress in this study, it cannot be determined exactly what may account for these correlations, although they could be attributed to the increased oxidative stress that the body is subjected to while practicing sport, this produces an acceleration of lipid peroxidation [18] and destroys the long chain fatty acids such as 22:6.

\section{Conclusions}

It can be concluded that 10 hours/week long-term physical football training modifies several fatty acids profiles in the erythrocyte membrane in young athletes. It was proved that C12:0, n-6 PUFA and $n-6 / n-3$ Index decreased while 22:6:3, LP 22:6:3 and LP 20:4:6 indexes increased.

The specific adaptations in the cell membrane to support the negative effect of ROS on the body of young players may reflect a positive consequence of aerobicanaerobic training. Significant correlations have been demonstrated between the training degree and the fatty acids indexes.

Although several healthy benefits of aerobic-anaerobic training have been found, antioxidant levels in both blood and diet are still necessary to obtain more consistent information and to reinforce the results obtained.

\section{Acknowledgements}

The authors sincerely thank the participants for taking part in this survey and for allowing us to get their erythrocyte samples. The authors acknowledge any financial interest or benefit arising from the direct application of this research.

The authors declare they have no actual or potential conflicts of interest.

\section{Ethical Statement}

The study was approved by the Ethics Committee of the University of Extremadura under the ethical guidelines of the Declaration of Helsinki of the World Medical Association (updated World Medical Assembly in Seoul 2008) for human research.

\section{References}

1. Nelson LD, Michael MC (2001) Lehninger principios de Bioquímica. Barcelona: Editorial Omega.

2. Riediger ND, Othman RA, Suh M, Moghadasian MH (2009) A systemic review of the roles of $n-3$ fatty acids in health and disease. J Am Diet Assoc 109: 668-679.

3. Woodside JV, Kromhout D (2005) Fatty acids and CHD. Proc Nutr Soc 64: 554-564.

4. Kromhout D, Giltay EJ, Geleijnse JM, Alpha Omega Trial Group (2010) n-3 fatty acids and cardiovascular events after myocardial infarction. N Engl J Med 363: 2015-2026.

5. Wakil SJ, Abu-Elheiga LA (2009) Fatty acid metabolism: target for metabolic syndrome. J Lipid Res 50: 138-143.

6. Tian T, Zhao Y, Huang Q, Li J (2016) n-3 Polyunsaturated Fatty Acids Improve Inflammation via Inhibiting Sphingosine Kinase 1 in a Rat Model of Parenteral Nutrition and CLP-Induced Sepsis. Lipids 51: 271-278.

7. Albert CM, Campos H, Stampfer MJ, Ridker PM, Manson $\mathrm{JE}$, et al. (2002) Blood levels of long-chain n-3 fatty acids and the risk of sudden death. N Engl J Med 346: 1113-1118.

8. Arab L (2003) Biomarkers of fat and fatty acid intake. J Nutr 133: 925-932.

9. Dayton S, Hashimoto S, Dixon W, Pearce ML (1966) Composition of lipids in human serum and adipose tissue during prolonged feeding of a diet high in unsaturated fat. J Lipid Res 7: 103-111.

10. Hodson L, Eyles HC, McLachlan KJ, Bell ML, Green TJ, et al. (2014) Plasma and erythrocyte fatty acids reflect intakes of saturated and $n-6$ PUFA within a similar time frame. $J$ Nutr 144: 33-41.

11. Smedman AE, Gustafsson IB, Berglund LG, Vessby BO (1999) Pentadecanoic acid in serum as a marker for intake of milk fat: relations between intake of milk fat and metabolic risk factors. Am J Clin Nutr 69: 22-29.

12. Mrakic-Sposta S, Gussoni M, Porcelli S, Pugliese L, Pavei $G$, et al. (2015) Training effects on ROS production determined by Electron Paramagnetic Resonance in master swimmers. Oxid Med Cell Longev 2015: 804794.

13. Pingitore A, Lima GP, Mastorci F, Quinones A, lervasi G, et al. (2015) Exercise and oxidative stress: potential effects of antioxidant dietary strategies in sports. Nutrition 31: 916922. 
14. Cheeseman $\mathrm{KH}$, Slater TF (1993) An introduction to free radical biochemistry. Br Med Bull 49: 481-493.

15. Halliwell B, Cross CE (1991) Reactive oxygen species, antioxidants, and acquired immunodeficiency syndrome. Sense or speculation? Arch Intern Med 151: 29-31.

16. Catalá A (2013) Five Decades with Polyunsaturated Fatty Acids: Chemical Synthesis, Enzymatic Formation, Lipid Peroxidation and Its Biological Effects. Journal of Lipids 2013: 1-19.

17. Yoshida Y, Umeno A, Shichiri M (2013) Lipid peroxidation biomarkers for evaluating oxidative stress and assessing antioxidant capacity in vivo. J Clin Biochem Nutr 52: 9-16.

18. Sumikawa K, Mu Z, Inoue T, Okochi T, Yoshida T, et al. (1993) Changes in erythrocyte membrane phospholipid composition induced by physical training and physical exercise. European Journal of Applied Physiology and Occupational Physiology 67: 132-137.

19. Goodpaster BH, He J, Watkins S, Kelley DE (2001) Skeletal muscle lipid content and insulin resistance: evidence for a paradox in endurance-trained athletes. $\mathrm{J}$ Clin Endocrinol Metab 86: 5755-5761.

20. Nikolaidis MG, Mougios V (2004) Effects of exercise on the fatty-acid composition of blood and tissue lipids. Sports Med 34: 1051-1076.

21. Arsic A, Vučić V, Tepšić J, Mazić S, Djelić M, et al. (2012) Altered plasma and erythrocyte phospholipid fatty acid profile in elite female water polo and football players. Appl Physiol Nutr Metab 37: 40-47.

22. Tepsic J, Vucic V, Arsic A, Mazic S, Djelic M, et al. (2013) Unfavourable plasma and erythrocyte phospholipid fatty acid profile in elite amateur boxers. Eur J Sport Sci 13: 414421.

23. Stølen T, Chamari K, Castagna C, Wisløff U (2005) Physiology of soccer: an update. Sports Med 35: 501-536.

24. Bangsbo J (2014) Physiological demands of football. Sports Science Exchange 27: 1-6.

25. Rollo I (2014) Carbohydrate: The football fuel. Sports Science Exchange 27: 1-8.

26. Booth M (2000) Assessment of physical activity: an international perspective. Research Quarterly for Exercise \& Sport 71: 114-120.

27. Esparza F (1993) Manual de Cineantropometría. Pamplona: (GREC) FEMEDE.

28. Porta J (1995) Valoración de la composición corporal. Análisis crítico y metodológico. Parte I. Car News 7: 4-13.

29. Lepage G, Roy CC (1986) Direct transesterification of all classes of lipids in a one-step reaction. J Lipid Res 27: 114120.
30. Halliwell B, Gutteridge JM (1985) Free Radicals in Biology and Medicine. Oxford: Clarendon Press.

31. Dise CA, Goodman DB, Rasmussen H (1980) Definition of the pathway for membrane phospholipid fatty acid turnover in human erythrocytes. J Lipid Res 21: 292-300.

32. Garaulet M, Pérez-Llamas F, Pérez-Ayala $M$, Martínez P, de Medina FS, et al. (2001) Site-specific differences in the fatty acid composition of abdominal adipose tissue in an obese population from a Mediterranean area: relation with dietary fatty acids, plasma lipid profile, serum insulin, and central obesity. Am J Clin Nutr 74: 585-591.

33. Helge JW, Wu BJ, Willer M, Daugaard JR, Storlien LH, et al. (2001) Training affects muscle phospholipid fatty acid composition in humans. J Appl Physiol (1985) 90: 670-677.

34. Takkunen M, Agren J, Kuusisto J, Laakso M, Uusitupa M, et al. (2013) Dietary fat in relation to erythrocyte fatty acid composition in men. Lipids 48: 1093-1102.

35. Heyward H (2008) Advance fitness assessment and exercise prescription. Madrid: Medica Paramericana.

36. Willmore JH, Costill DL (2007) Fisiología del esfuerzoy del deporte. (6 $6^{\text {th }}$ édn), Barcelona: Paidotribo.

37. Miranda R, Antunes H, Pauli JR, da silva A (2013) Effects of 10-week soccer training program on anthropometric, psychological, technical skills and specific performance parameters in youth soccer players. Science \& Sports 28: 81-87.

38. Andersson A, Sjödin A, Olsson R, Vessby B (1998) Effects of physical exercise on phospholipid fatty acid composition in skeletal muscle. Am J Physiol 274: 432-438.

39. Mori TA, Bao DQ, Burke V, Puddey IB, Beilin LJ (1999) Docosahexaenoic acid but not eicosapentaenoic acid lowers ambulatory blood pressure and heart rate in humans. Hypertension 34: 253-260.

40. Tepsic J, Vucic V, Arsic A, Blazencic-Mladenovic V, Mazic S, et al. (2009) Plasma and erythrocyte phospholipid fatty acid profile in professional basketball and football players. Eur J Appl Physiol 107: 359-365.

41. Mozaffarian D, Wu JH (2011) Omega-3 Fatty Acids and Cardiovascular Disease: Effects on Risk Factors, Molecular Pathways, and Clinical Events. J Am Coll Cardiol 58: 20472067.

42. Yang LG, Song ZX, Yin H, Wang YY, Shu GF, et al. (2016) Low n-6/n-3 PUFA Ratio Improves Lipid Metabolism, Inflammation, Oxidative Stress and Endothelial Function in Rats Using Plant Oils as n-3 Fatty Acid Source. Lipids 51: 49-59.

43. Shigenaga MK, Hagen TM, Ames BN (1994) Oxidative damage and mitochondrial decay in aging. Proc Natl Acad Sci U S A 91: 10771-10778. 